\title{
PULSAR EMISSION BEAMS AND PROFILES IN AN INVERSE-COMPTON SCATTERING MODEL
}

\author{
G. J. QIAO \\ Center of Astronomy and Astrophysics, CCTS (World Laboratory) and Department of Geophysics, Peking University
}

\begin{abstract}
The vast majority of pulsar profiles at meter wavelengths are dominated by core components (Rankin 1983, 1990; Lyne and Manchester 1988), but in the usual polar cap models of pulsar emission, it is difficult to get central beam or "core" radiation. In this paper, we present a calculation for both the "core" and hollow "cone" emission beams, as well as model pulse profiles in an inverse-Compton scattering (ICS) model. Both "core" and hollow "cone" emission beams are obtained naturally in the calculations. Examples of pulse profiles of pulsars at different radio frequencies are presented.

The theoretical shapes of the pulse profiles agree very satisfactorily with actual observations, which means that the mechanism suggested here may be the actual one.
\end{abstract}

\section{Introduction}

The differing properties of core and conal emission discussed by Rankin (1983) have been confirmed by Lyne and Manchester (1988). Their work provides a very firm basis for emission models. The conal emission has been studied extensively, both observationally and theoretically. Only a few authors, such as Gil (1985), Qiao (1988a), Wang et al. (1989), and Beskin et al. (1988) have presented models for core emission. In this paper, we present a calculation for both the core and conal emission beams, as well as model average emission profiles according to an inverse-Compton scattering model (Qiao 1988a). In the calculation, the picture develops naturally that a central or "core" radiation beam is surrounding by one or two hollow conal radiation beams, which is just what Rankin (1983) concluded from the observations. We find that the beam pattern can exhibit the various pulse profiles that have been observed.

\section{The inverse-Compton scatter- ing (ICS) model}

The basic idea of the ICS model is that the high energy particles are moving along the magnetic field lines and scattering the low frequency photons. The low frequency photons are produced by the sparking in the inner gap. The outgoing photons from the scattering are the photons which are observed. The beam pattern is produced by the scattering.

\section{The low frequency wave}

When a spark takes place in the inner gap, its intensity may fluctuate as the plasma increases (decreases) and the potential drop decreases (increases). The fluctuation time scales should be $\sim 10 \mu$ s (Ruderman and Sutherland 1975). Similar time variations have been observed in individual pulsar pulses (Hankins 1971, 1972). This fluctuation would be associated with a very strong radio wave, the period of it would be $\sim 10 \mu s$, and the frequency $\omega \sim 6 \times 10^{5} \mathrm{~s}^{-1}$

\section{High energy particles}

Xia et al. (1985), Daugherty and Harding (1986, 1989) find that the Compton energy losses of the pairs are higher than the curvature losses of the primaries, and the pairs lose their energy in ICS with the thermal photons in the gap. This energy loss limits the Lorentz factor $\gamma$ of the $\mathrm{e}^{ \pm}$. For higher temperatures, $\gamma$ is lower. In the calculation of this paper, $\gamma$ is taken between $10^{2}$ and $10^{3}$.

\section{ICS process and outgoing photons}

For most pulsars $B \ll B_{\mathrm{q}}$ close to or far from the surface of the star, $B_{\mathrm{q}}=4.414 \times 10^{13} \mathrm{G}$. In the electron rest frame

$$
\omega^{\mathrm{R}}=\gamma \omega\left(1-v / c \cos \theta_{\mathrm{i}}^{\mathrm{L}}\right) \leq 2 \gamma \omega^{\mathrm{L}} \ll \omega_{\mathrm{B}}
$$

where $\theta_{\mathrm{i}}^{\mathrm{L}}\left(\theta_{\mathrm{i}}^{\prime \mathrm{L}}\right)$ is the angle between the incoming (outgoing) photons and the direction of the magnetic field. $\omega_{\mathrm{B}}=e B / m c$ is the magnetic gyrofrequency. " $R$ " and " $L$ " denote the rest frame of the electron and the laboratory frame, respectively. In this case, the total cross section for ICS in the laboratory frame is as follows (Qiao 1988a) 


$$
\begin{aligned}
\operatorname{rr}_{\mathrm{L}}(1) & =\frac{\sin ^{2} \theta_{\mathrm{i}}\left(1-\beta \cos \theta_{\mathrm{i}}\right)}{\gamma^{2}\left(1+\beta \cos \theta_{\mathrm{i}}\right)^{2}} \sigma_{+\mathrm{h}} \\
& = \begin{cases}0 & \text { for } \theta_{\mathrm{i}}=0 \\
\gamma^{-2} \sigma_{+h} & \text { for } \theta_{\mathrm{i}}=\pi / 2\end{cases} \\
\sigma_{\mathrm{T}}(2) & =0
\end{aligned}
$$

where " 1 " (" 2 ") is the component of the polarization vector of the incoming photons-that is the electric vector parallel (perpendicular) to the incoming plane. As $\gamma \gg 1, \gamma h \omega \ll m_{\mathrm{e}} c^{2}$ and $\theta^{\prime} \sim 0$, we have (Qiao 1988b)

$$
\hbar \omega^{\prime}=2 \gamma^{2} \hbar \omega\left(1-\frac{v}{c} \cos \theta_{i}\right),
$$

where $\hbar \omega^{\prime}$ is the energy of the outgoing photons and $v$ is the velocity of the pairs.

\section{Calculation of the emission beams and pulse profiles}

For a dipole magretic field line, we have

$$
\rho=r_{\mathrm{e}} \cos ^{2} \varphi
$$

At point $(\rho, \varphi), \theta$ and $\varphi$ (see figure 1 ) we have the

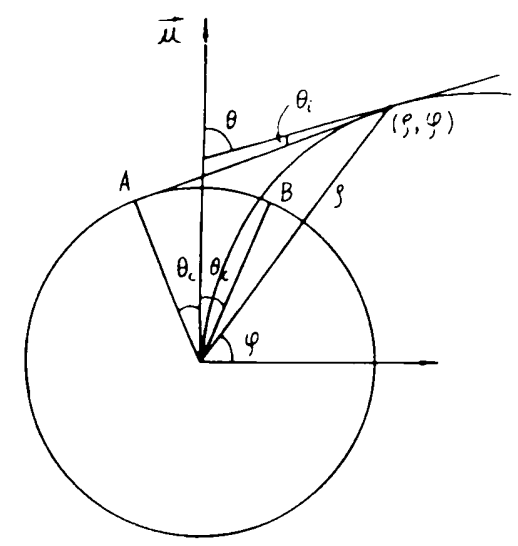

Figure 1 The geometry of ICS. As the sparking takes place around the cap, the low frequency wave is emitted from point $A$ to point $B$ equivalently. The low frequenc waves are scattered by the pairs moving along the magne. $1 \mathrm{c}$ field lines. Line $\mathrm{MN}$ is the tangent line of the magnetic held line at point $\mathrm{N}(\rho, \varphi)$. MN is the direction of the observed emission.

following relationship (Qiao 1988a)

$$
\tan \varphi=\frac{1}{4}\left(3 \cot \theta \pm \sqrt{9 \cot ^{2} \theta+8}\right) .
$$

The angle $\theta_{\mathrm{i}}$ for the emission at point $\mathrm{A}$ is

$$
\tan \theta_{\mathrm{i}}=\frac{\rho \sin c-R \sin b}{R \cos b+\rho \sin c}
$$

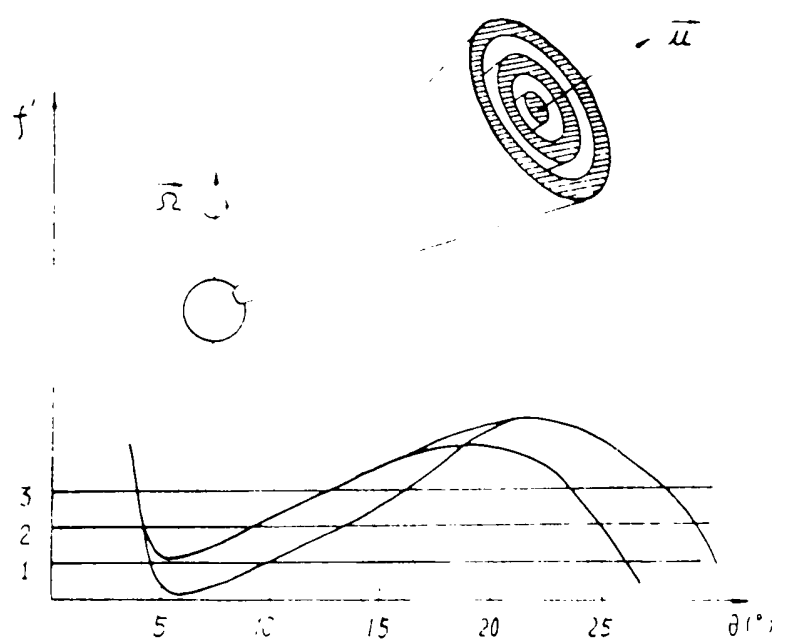

Figure 2 The observed radiation frequency $f^{\prime}$ as a function of $\theta$, and the equivalent radiation beam pattern. Note that the shape of $f^{\prime}-\theta$ is a function of pulsar period $P$ and the value of $\xi$. The patterns of the radiation beams vary with the observing frequency.

where $R$ is the radius of the neutron star, $R \sim 10^{6} \mathrm{~cm}$, $b=\pi-\theta_{\mathrm{c}}-\theta, c=\theta+\varphi-\pi / 2$, and $\theta_{\mathrm{c}}=(\Omega R / c)^{1 / 2}=$ $(2 \pi R / P c)^{1 / 2}$, where $P$ is the period of the pulsar. For low frequency radiation from point $B$, the angle $\theta_{\mathrm{i}}$ is

$$
\tan \theta_{\mathrm{i}}=\frac{\rho \sin c^{\prime}-R \sin b^{\prime}}{R \cos b^{\prime}+\rho \sin c^{\prime}},
$$

where $b^{\prime}=\theta-\theta_{c}$ and $c^{\prime}=\theta+\varphi$.

The angle $\theta$ is just the angle between the radiation direction and magnetic axis. From eqs.(2)(5), we can find $\omega^{\prime} \sim \theta$ for each $r_{\mathrm{e}}$ (for example, $\left.r_{\mathrm{e}}=R_{\mathrm{LC}}, 10 R_{\mathrm{LC}} \ldots\right)$, where $R_{\mathrm{LC}}$ is the radius of the light cylinder.

In our calculation, we assume that

$$
\gamma=\gamma_{0}(1-\xi \rho) \text {. }
$$

Here $\xi$ represents the energy loss of the pairs and the effects of the bending of the magnetic field, which is lifferent from pulsar to pulsar.

For different magnetic inclination $\alpha$ and impact angle $\beta$, the pulse profiles are different. We have

$$
\cos \theta=\cos \alpha \cos (\alpha+\beta)+\sin \alpha \sin (\alpha+\beta) \cos \phi
$$

Here $\phi$ is the longitude angle. In the normal polarcap model, the maximum rate of position angle swing occurs when the observer is on the magnetic meridian and is given by (Lyne and Manchester 1988)

$$
(d \psi / d \phi)_{\mathrm{m}}=\sin \alpha / \sin \beta .
$$

The value of $(d \psi / d \phi)_{\mathrm{m}}$ is given from the observations. In our case, the radiation from different magnetic lines is pressed (see figure 2), so eq.(8) is not always correct. We still, however, take it as a reference. 


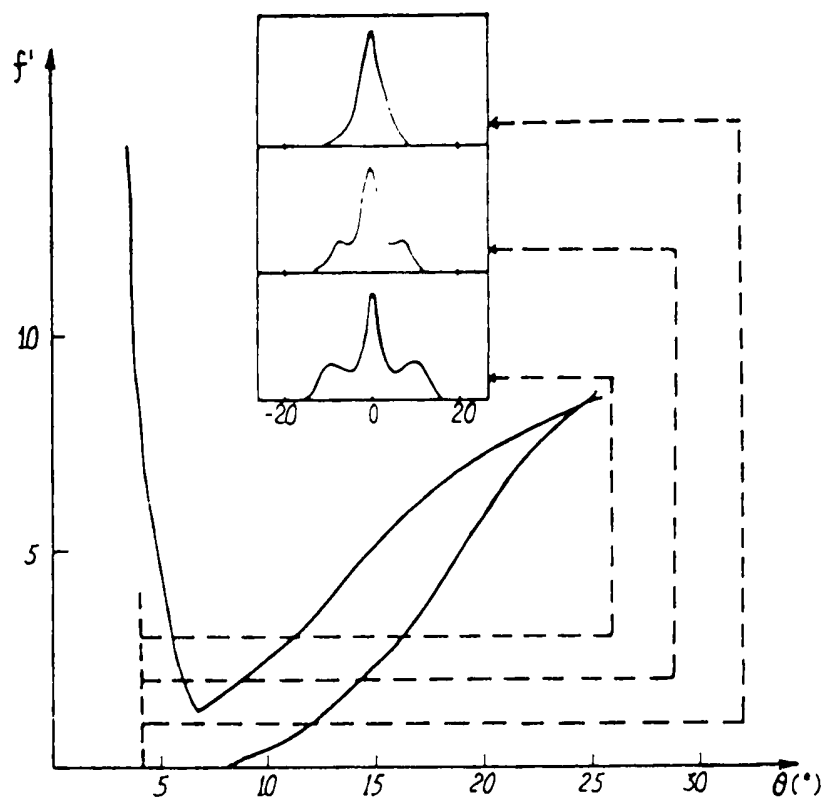

Figure 3 Observed frequency $f^{\prime}$ as a function of $\theta$ and the pulse profiles observed at different frequencies ( $c f$. PSR 1642-03; see Sieber et al. 1975).

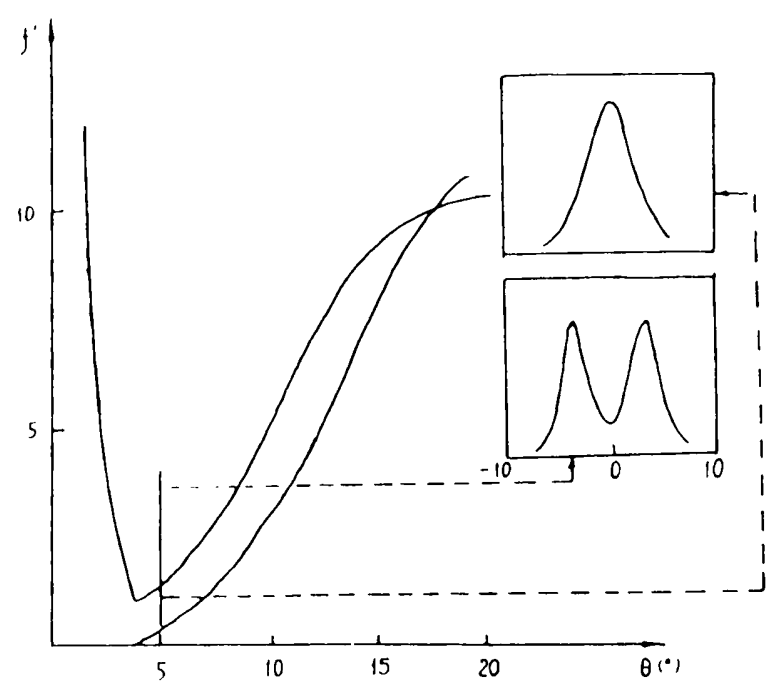

Figure 5 Observed frequency $f^{\prime}$ as a function of $\theta$ and the pulse profiles observed at different frequencies ( $c f$. PSR 0834+06; see Kuz'min et al. 1986).

The results of the calculations are shown in figure 3 to 7 .

The characteristics of the radiation beams are as follows:

1. The width of the "core" beam increases as the period of the pulsar is decreases.

2. The shape of the hollow cone beam is not the same from pulsar to pulsar, rather it is sensitive to the energy loss of the pairs and/or the curvature of the magnetic field lines-which in turn are sensitive to the value of $\xi$ in eq.(6).

3 . The radiation beam is not the same at different frequencies; this is especially true for the conal beam.

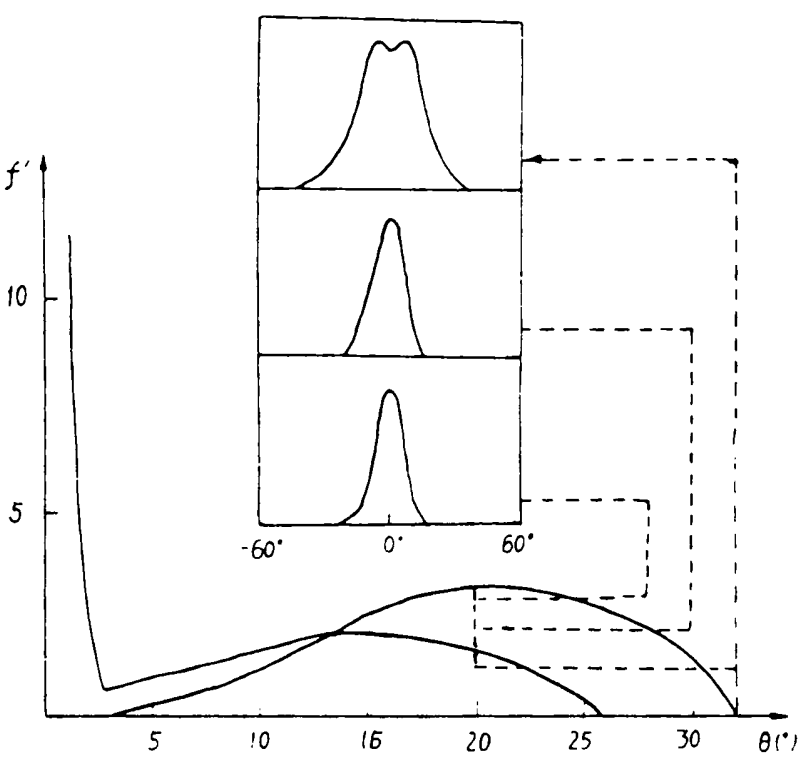

Figure 4 Observed frequency $f^{\prime}$ as a function of $\theta$ and the pulse profiles observed at different frequencies ( $c f$. PSR 0809+74; see Izvekova et al. 1979).

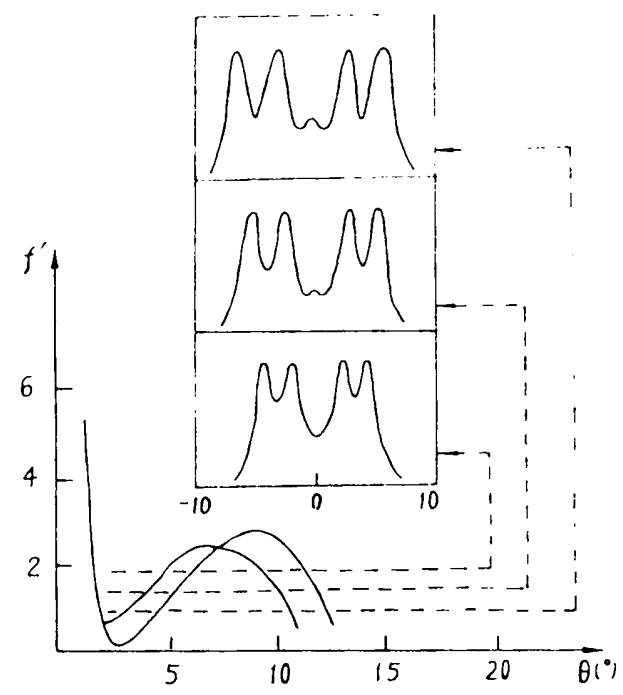

Figure 6 Observed frequency $f^{\prime}$ as a function of $\theta$ and the pulse profiles at different frequencies (cf. PSR $1237+25$; see Bartel et al. 1982).

The form of the average profiles is determined by the shape of the beams as well as by the magnetic inclination $\alpha$ and impact angle $\beta$. Some examples of pulse profiles at different frequencies are presented in figures 3 to 7 , including a conal single profile (PSR 0809+74), core single profiles (PSR 1933+16, PSR 1642-03), a conal double profile (PSR 0834+06), a the multiple (five-component) profile (PSR $1237+25$ ). The model profiles agree very satisfactorily with the observations. 


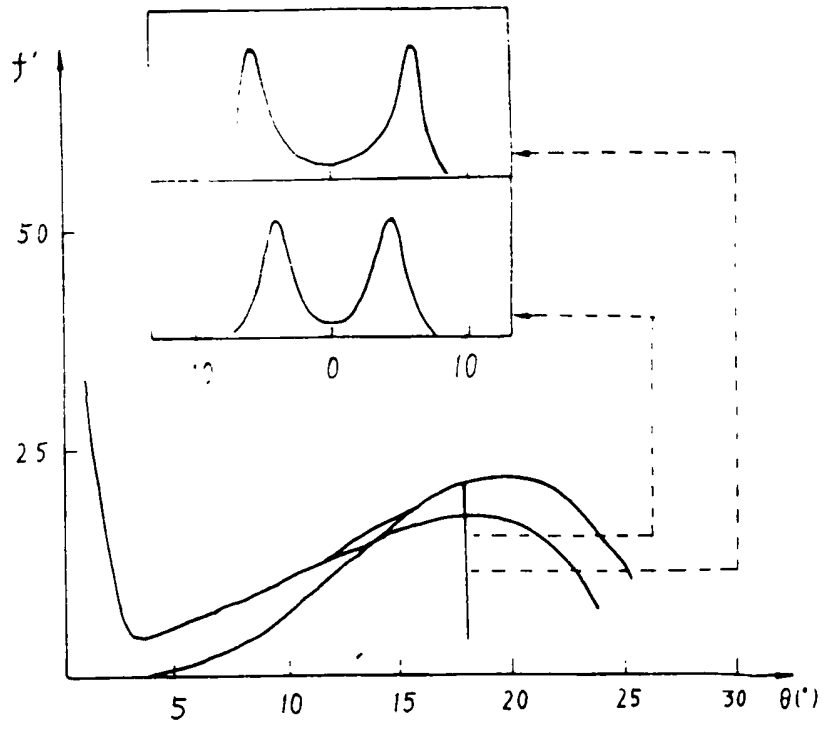

Figure 7 Observed frequency $f^{\prime}$ as a function of $\theta$ and the pulse profiles at different frequencies (cf. PSR 0525+21; see Gil et al. 1992).

\section{Conclusions}

1. The agreement above shows that the ICS model suggested here is not a coincidence, rather it appears to represent the actual physical circumstances which operate in pulsars.

2. Rankin (1983) has recognized the distinct properties associated with the respective "core" and "conal" components, such as the circumstance that core components may originate relatively close to the neutron star (Rankin 1990). Lyne and Manchester (1988) find that the observations are best described by a gradual change in emission characteristics from the core region to the outer edge of the emission beam, rather than two distinct emission processes. These points of view are supported by our calculations.

3. The ICS mechanism may also be responsible for the optical emission of pulsars (Qiao et al. 1986, Zhu and Qiao 1989).

4. The locations of emission for both the core and conal emission can be calculated within the framework of the ICS model (see the companion paper below, Qiao et al. 1991).

5. In the ICS model, the jumps of the linear position angle can be explained naturally.

6. Within the ICS model, three parameters $(\alpha, \beta, \xi)$ can be adjusted for each pulsar; all of them, however, can be decided via multi-frequency observations. A new catalog for $\alpha$ and $\beta$ would be obtained, which would be different from previous efforts.

Acknowledgments: We are grateful to Profs. J. M. Rankin, R. N. Manchester, and J. Gil for their very helpful discussions. The author thanks Mr. Y. D. Zhu and H. Chen for their useful suggestions, and also thanks Mr. C. G. $\mathrm{Li}$ for his help in preparing this work. This project was supported by the Natural Science Foundation of China. 